\title{
Graphite Pencil Drawn Lines: A Nanomaterial or Few Layer Graphene/Graphite Layered Structure
}

\author{
G.Sh. Shmavonyan ${ }^{1}$ and A.R. Mailian ${ }^{2}$ \\ ${ }^{1}$ National Polytechnic University of Armenia, Department of Microelectronics and Biomedical Devices, 105 Teryan str., Yerevan, \\ 0009, Armenia \\ ${ }^{2}$ Institute for Informatics, 1 P. Sevak str., 0014, Yerevan, Armenia
}

\begin{abstract}
A non-conventional method of obtaining few-layer graphene (FLG) is suggested, which comprises repeatedly rubbing bulk graphite against semiconducting and insulating substrates. The obtained graphene/graphite structures consisting of rubbed-off layers behave like a semiconducting material with through-thickness anisotropy of carrier mobility reaching to $3000 \mathrm{~cm}^{2} / \mathrm{V}$.sec at the surface. The structure surface demonstrates more ordered and oriented character containing FLG. The suggested method allows easily obtaining graphene with pencil directly on paper, as well as graphene-based electronic components and circuits on paper and other substrates, which could enable flexible and cheap electronics. The observed phenomenon is universal, does not depend on the substrate material and could find a widespread application.
\end{abstract}

Keywords—graphene; graphite; pencil; drawing

\section{INTRODUCTION}

Graphite thin films are extensively used in electronics (resistors, conducting layers in printed circuits, under-button contacts, etc.) so far. The interest toward their physical properties increased in recent past as a consequence of the discovery of fascinating properties in graphite single layers. In timeline, isolation of one-atom-thick layer of carbon was the last one of a triumphant series of obtaining various graphitebased low-dimensional materials, such as zero dimensional carbon fullerene [1], 1D-nanotube [2] and 2D-graphene [3], an atomically thick basic carbon plane. Graphene is the most practical structure in this series since its electronic properties are easy to control by external factors and it is convenient to utilize. FLG is a unique material with its own potential for device applications.[4-6] Mechanical exfoliation is still a reliable and simple method to obtain FLG flakes with high electronic quality. By this method a layered graphite crystal is rubbed against the substrate surface leaving nanoscale flakes attached to it. It is much like drawing by pencil. Then, single or few-layer flakes are picked up from rubbed off and attached to substrate surface set of flakes.[7,8] The whole trace or graphite thin structure or just the pencil drawn line as a material was disbelieved to have attractive electronic properties and because of that, perhaps, have been overlooked to be studied. On the other hand, we believed its micro-scale thickness ought to alter the behavior of electron gas and it could be an interesting object of investigation.

This pioneering report summarizes the experimental study of some basic properties of mechanically exfoliated graphite structures obtained from disordered graphite bulk by rubbing on different substrates.

\section{EXPERIMENT}

Samples of graphite thin structures were prepared by series of sequential rubbing parent graphite rods against the surface of insulating and semiconducting substrates along the same path (see inset of Figure 1). We commonly used pencil leads of H, HB, Fand B hardness, though all types of rods (highly oriented pyrolitic graphite, graphite used in electrical engineering and chemistry, etc.) showed the same behavior. As a substrate we mainly used paper for convenience though, again, all types of the substrates showed identical results.

In order to peel off layers, the parent graphite rod was pressed normal to the substrate surface with the pressure of $\geq 1 \mathrm{MPa}$, which is higher than the force required for cleaving off a flake with the thickness of several atomic layers from the bulk.[9] Actually drawing a line is a mechanical modification by combined and continuous operation of cleavingtransferring-pressing of flakes. Therefore, we call the drawn lines Combined Mechanical Modified (CMM) layers and the structures containing multiple CMM layers - CMM structures. The advantages of our preparation method lie in the easy way to realize and it avoids contamination or surface doping avoiding the contact with any other material.

The electrical resistance of the structures was measured by a conventional two-probe method, using gold contacts pressed on the surface of the structures.

Raman spectra were measured using a RENISHAW in Via Raman at excitation wavelength of $514 \mathrm{~nm}$, at room temperature. The Raman spectral resolution was better than 4 $\mathrm{cm}^{-1}$ and the incident power of the excitation laser light was $0.6 \mathrm{~W}$.

\section{RESULTS AND DISCUSSION}

Our experimental observations show that observed regularities and peculiarities presented hereafter originate from the surface or surface-adjacent stratum of the CMM structure. First, it was found that the CMM structures behave like a solid state material exhibiting a number of interesting physical properties common to layered fine structures. The carrier transport is dimensionality dependent. Namely, the electrical resistance taken from the structure surface undergoes drastic change with the number of cleaved and transferred layers, i.e. with the thickness of the CMM structure 
(Figure 1a). Typically, the conductance became clearly detectable after a couple of rubbings. Take for example, samples presented in Figure 1a. At the same pressure, and with the same graphite rod, layers on glass, paper and ceramic substrates exhibited conductance at the $2^{\text {nd }}$ rubbing or transfer, while layers on $\mathrm{ZnO}$ ceramic and plastic substrates at the $3^{\text {rd }}$ rubbing.

During a few following rubbings the resistance dropped $\sim 1000$ times, from several Mohm to several hundred ohm reaching low-resistance plateau $(\mathrm{N}>10)$. Note, that the resistance of the structures never reached that of the bulk. Such a behavior is common to the structures on different substrates and different graphite rods, and hence unambiguously reflects the intrinsic properties of graphite (Figure 1a).

Following the growth of the structure under optical microscope, the pre-conduction structure was seen as a field of casual and rare spread spots or wormlike threads (paper substrate) of several micrometer sizes. This picture should mean that initial rubbed off flakes merely filled pores of the substrate (layers 1 and 2, Figure 1b). The ground conducting layer and structures appearing at further few rubbings $(\mathrm{N}=2 \div 5$ in Figure 1) may have been shaped by chain of randomly stacked flakes bridging the neighboring graphite filled pores.

Disorder in these layers should strongly limit the conductance, since the sizes of flakes are close to mean free path of electrons and therefore boundary scattering is extremely high. Thus, a CMM structure should consist of graphite isles attached to the substrate and continuous and conducting layers sandwiched above. Hereafter we will examine the behavior of conducting layers shaped at each rubbing and the whole structure consisting of individual conducting layers. The rubbing numeration which is the same for transfer, will be kept unchanged as shown in Figure 1b.

For characterizing the obtained structure, as well as for understanding the observed regularities one needs to know the thickness of the structure. The thickness of $\mathrm{N}^{\text {th }}$ transferred layer, as and the total thickness of the conducting stratum in total structure containing $N$ transferred layers, $d_{N}=$ is possible to calculate measuring the abraded volume of the parent graphite rod during rubbing (inset in Figure $1 \mathrm{a}, \mathrm{b}$ )[12]. With a rod having round cross section, the thickness of a single rubbed off layer can be calculated by $=D l / 4 L$, where lis the change in the rod length, $D$ - diameter of the rod, $\mathrm{L}$ - the length of transferred layer (inset in Figure 1). As the thickness of the whole structure increases with successive steps of rubbing, the upper transferred individual conducting layers progressively get thinner (Figure $2 \mathrm{a}$ ). The thickness of $\mathrm{N}^{\text {th }}$ transferred layer is estimated by $d d$ inset in Figure 2a. For $\mathrm{N}=20 \div 30$ corresponding to the region of plateau on resistance vs. $N$ curve (Figure 1a), is estimated to range from $\sim 1$ to $8 \mathrm{~nm}$ depending on the surface roughness of substrates and graphite hardness. This value is quite reasonable for as the structure becomes thicker the same bonding strength between crystal planes in both grown structure and parent graphite bulk should limit the maximum thickness of a single peeled off flake from both sides. Thus, due to tightly packed and ordered stacking of upper layers in the CMM structure, the curve of thickness vs. number of transferred layers $\left(d_{N}\right)$ tends to saturate since $d_{N}=$, like the case shown in Figure 2a.

Next characteristic parameter, the carrier mobility is deduced from the measured electrical resistance of the structure by

$$
\mu_{N}=\operatorname{a/en} \rho_{N}=a / e n w R_{N} d_{N}
$$

where $e$ is the charge of an electron, $a$ and $w$ are the length and the width of the structure segment between measuring contacts, respectively, $n$ - the bulk carrier density and $R_{N}$ - the electrical resistance taken from the surface of the CMM structure containing $N$ transferred layers. For instance, for the sample presented in Figure 2 quantitative assess revealed enhancement in the mobility by a factor of $10 \mathrm{~s}$ with respect to the ground conducting layer with $\mathrm{N}=3$ (Figure $2 \mathrm{~b}$ ). However, one can see apparent discrepancy between this value and sharp drop in the structure resistance. Consequently, this suggests anisotropy in the stacking geometry and the mechanism of carrier transport should be different through the structure thickness[13]. Thus, to obtain accurate values for mobility it is necessary to take into account this aspect.

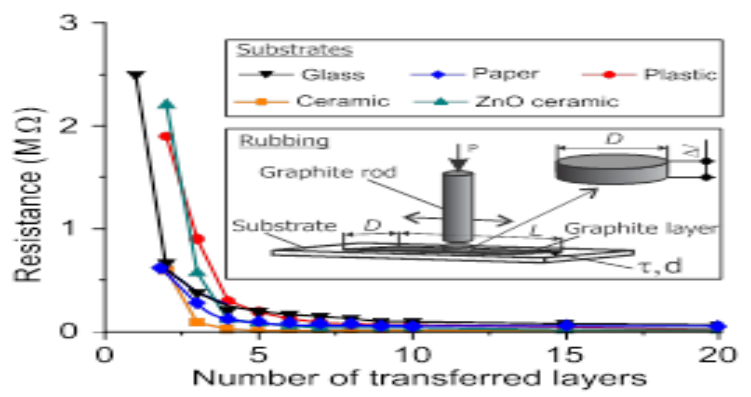

a)

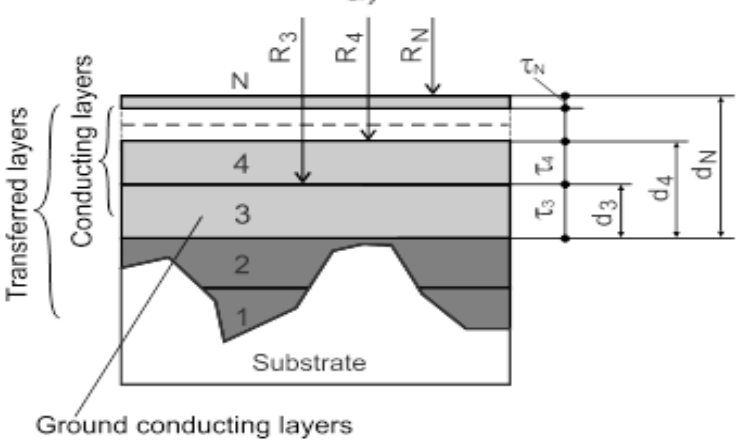

b)

FIGURE I. A) TYPICAL DEPENDENCE OF THE ELECTRICAL RESISTANCE OF THE CMM STRUCTURES ON THE NUMBER OF TRANSFERRED LAYERS FOR DIFFERENT INSULATING SUBSTRATES AND SEMICONDUCTING ZnO At Applied Pressure Of P $5 \mathrm{MPa}$. INSET SCHEMATICALLY ILLUSTRATES THE FABRICATION PROCESS OF A CMM STRUCTURE, WHERE TWO ARROWS INDICATE THE DIRECTION OF THE MOVEMENT OF BULK GRAPHITE ROD DURING RUBBING. B) SCHEMATIC PRESENTATION OF PACKING OF TRANSFERRED LAYERS IN THE CMM. ON THIS EXAMPLE CONDUCTING TAKES PLACE AT THE $2^{\text {nd }}$ RUBBING OR

TRANSFER. VERTICAL ARROWS SHOW THE SURFACES FROM WHICH THE RESISTANCE WAS MEASURED 


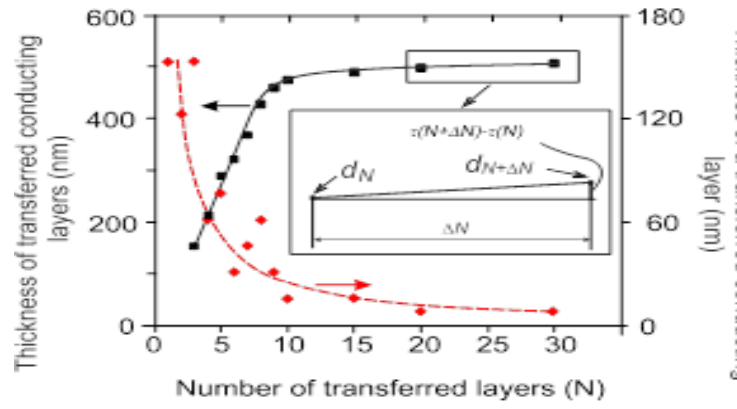

a)

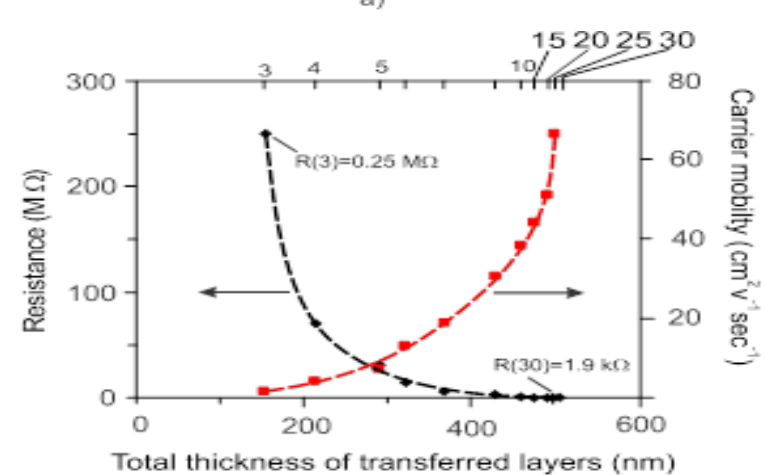

b)

FIGURE II. A) TYPICAL DEPENDENCE OF TOTAL THICKNESS OF THE CONDUCTING LAYER IN THE CMM STRUCTURE (BLACK LINE) AND THE THICKNESS OF SINGLE RUBBED OFF LAYER (RED LINE) ON THE NUMBER OF THE TRANSFERRED LAYERS ON PAPER. THE INSET ILLUSTRATES THE CALCULATION OF THICKNESS OF ONE RUBBED OFF LAYER. B) TYPICAL DEPENDENCE OF RESISTANCE OF CMM STRUCTURE ON TOTAL THICKNESS OF CONDUCTING LAYER AND THE NUMBER OF TRANSFERRED LAYERS COMPARED WITH CARRIER MOBILITY

To identify the conducting mechanism in the CMM structure, the temperature $(\mathrm{T})$ behavior was studied as well (Figure 3). As can be seen, the electrical resistance of the CMM structure decreases with the increase of the temperature. There is an obvious difference in the T-dependence between thick, i.e. more conductive and thinner (less conductive) samples. Note, that no metallic-like behavior was observed.

To understand the nature of the electrical conduction in these structures we consider a semilog plot of the inverse temperature dependence of inverse resistance for a representative samples (Figure 3). In all cases we find that $\ln (1 / R)$ has a similar functional dependence on $T^{-1}$ characterized by three regions with distinct slopes extracted from the plots in Figure 3 a.

These experimental results expose semiconducting nature of the CMM structures and provide clear evidence for layered and ordered stacking of the CMM structures. Electronic states responsible for linear slopes in $\ln (1 / R)$ vs. $1 / T$ plot do not exist neither in single-layer nor in bulk graphite. They originate from modification of continuous and linear . and . bands at $\mathrm{K}$ point of Brillouin zone under influence of interaction between basic layers.[11] So, the CMM structures demonstrate quasi two-dimensionality of carrier transport [1215], with an alternate conducting mechanism. Also, such Tbehavior lends support for the model of through-thickness anisotropy.
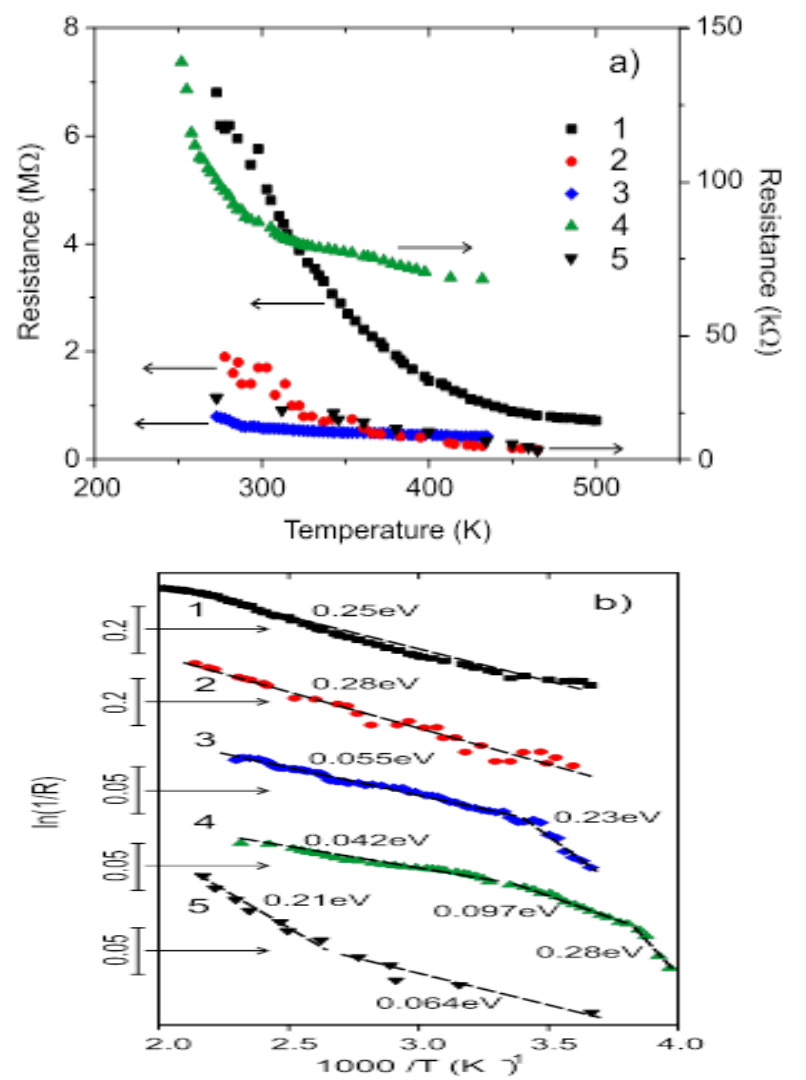

FIGURE III. A) TEMPERATURE DEPENDENCE OF ELECTRICAL RESISTANCE OF VARIOUS CMM STRUCTURES ONA PAPER SUBSTRATE. THE STRUCTURE RESISTANCE DECREASES BY INCREASING THE ORDER OF CURVES.B) THE SAME DEPENDENCE IN SEMILOG PLOT OF THE INVERSE TEMPERATURE. THE BARS ON THE LEFT SIDE PRESENT THE SCALE. THE NUMBERS IN EV PRESENT THE ACTIVATION ENERGIES CALCULATED FOR LINEAR PARTS

Further on, as the surface layers of any ordered graphitic structure are totally screened from the substrate due to huge anisotropy in in-plane and out-of-plane conductivity and sources of disorder are reduced significantly at a layer surface [16-18], we argue that mainly the resistance of the top layer is measured from the contacts pressed on surface. Our observation and measurements too speak about totally independent behavior of transferred layers: the resistance of bottom layers did not change with rubbing upper layers. In addition, concerning the measurements of resistance from the surface of the CMM structure, the inter-layer screening length to electric field in thin graphite is estimated to be $1.2 \mathrm{~nm}$, which is about four monolayer thick.[19] This leads to assume over again that the contribution of the surface structure in carrier transport is crucial for the CMM structures. 
Explicit features of the layered CMM structure is expected to reveal by Raman spectroscopy since it is sensitive to the surface, layer number and structural and stacking order.[1922]. Raman spectra of the CMM structures are shown in Figure 4, which allow us to conclude that FLG $(\mathrm{N}=2 \div 4)$ is formed on the CMM structure surface.

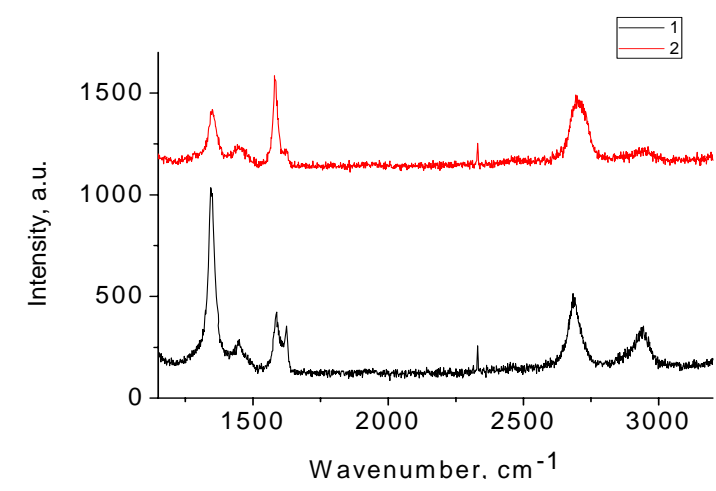

FIGURE IV. RAMAN SPECTRA OF FEW LAYER GRAPHENE/GRAPHITE STRUCTURE AT TWO DIFFERENT POSITIONS ON THE SURFACE AT EXCITATION WAVELENGTH OF $514 \mathrm{NM}$

Besides, following visually the patterning of the CMM structure, its surface gets shine when the resistance reaches the plateau (Figure 1) and the friction between the CMM structure and parent bulk rod decreases significantly. Usually this means that the surface of the structure becomes ordered and crystallograpically oriented $[23,24]$. We observed also that the light reflected from the surface of the CMM structure is polarized normal to the structure plane. This testifies to the presence of a self-organized (due to the friction during rubbing), ordered and crystallographically oriented surface. $[25,26]$

Thus, FLG flakes shape a continuous lamina on the surface of the graphite layers.

Having these results one can explain the discrepancy in the value of mobility calculated above. As the intrinsic disorder is substantially reduced through the thickness of the CMM structure having been transformed into an ordered and stacked crystal structure at the surface, we can use $\mu_{N}=a /$ enw $R_{N} \tau_{N}$ instead of (1).Taking $\tau_{N}=1.4 \mathrm{~nm}$, the thickness of 4-fold graphite monolayer for $N=20 \div 30$ and assuming that $n \sim 10^{18}$ $\mathrm{cm}^{-3}$, for experimentally measured $R_{N}=1.9 \mathrm{k} \Omega$, the calculation yields the mobility value of $\mu \sim 3000 \mathrm{~cm}^{2} \mathrm{~V}^{-1} \mathrm{sec}^{-1}$. Now, this value matches well with the observed decrease in the electrical resistance of $\sim 1000$ times.

\section{CONCLUSION}

In summary, it is found that the layered structure obtained by combined mechanical modification or repeatedly rubbing of the graphite bulk exhibits peculiar physical properties, such as:a) thetop layer of the structure containing mechanically transferred layers is orderly stacked and crystallographically oriented perpendicular to the structuresurface, b) thecarrier mobility is anisotropic through the thickness of the structure with the highest value of $\sim 3000 \mathrm{~cm}^{2} / \mathrm{V} \cdot \mathrm{sec}$ at the top of the structure.

The overall results testify to a structure promising to obtain importance in achieving selective conductivity and high carrier mobility in the graphite/graphene system.

The suggested method allows easily obtaining graphene with pencil directly on paper, as well as designing graphenebased electrical components and circuits on paper and other substrates, which could enable flexible and cheap electronics. The observed phenomenon is universal, does not depend on the substrate material and could find a widespread application.

\section{REFERENCES}

[1] Kroto H.W., Heath J.R., O’Brien S.C., Curl R.F., Smalley P.E. Nature 318, 162-163 (1985).

[2] Iijima S. Nature (London) 354, 56-58 (1991).

[3] Novoselov K.S.,Geim A.K., Morozov S.V., Jiang D.; Zhang Y., Dubonos S.V.,Crigorieva I.V., Firsov A.A.Science 306, 666 (2004).

[4] Bunch J.S., Yaish Y., Brink M., Bolotin K., McEuen P.L. Coulomb Nano Letters 5 , 287-290, (2005).

[5] Wu H., Yang B.J., Zong B.Y., Sun H., Shen Z. X., Feng Y P. J. Mater. Chem. 14, 469-477 (2004).

[6] Zhang B., Small J.P., Pontius W.V., Kim P. Appl. Phys. Lett., $\underline{86}$, 073104-073104-3 (2005).

[7] Novoselov K.S., Jiang D., Schedin F., Booth T. J., Khotevich V.V., Morozov S.V., Geim A.K. Proceedings of Nat. Acad. Sc., 102, No. 30, 10451-10453 (2005)

[8] Frank I.W., Tonenbaum D.M,Zande van der A.M., McEuen P.L. J. Vac. Sci. Technol. B25(6), 2558-2561 (2007).

[9] Zhang Y.B., Small J.P., Pontius W.V., Kim P. Appl. Phys. Lett. 86, 073104 (2005).

[10] Woolf L.D, Streckert H.H. The Physics Teacher, 34, 440 (1996).

[11] Castro Neto A.H., Guinea F., Peres N.M.R.,Novoselov K.S., Geim A.K Reviews of Modern Physics, 81, 109-162 (2009).

[12] Shmavonyan G., Mailian A., Mailian M. Thessaloniki, Greece, Abstract Book, p.280 (2013).

[13] Barzola-Quiquia J., Yao J.-L., Rodiger P., Schindler K., Esquinazi P. Phys. Stat. Sol., (a) 205, 12, 2924-2933 (2008).

[14] Vasko, F.T., Ruzhii, V. Phys. Rev. Lett.76, 233404 (2007)

[15] Shao Q., Liu G., Teweldebhan, D.,Balandin A.A. Appl. Phys. Lett., 92 202108 (2008).

[16] Garcia N., Esquinazi P., Barzola-QuiquiaJ., Dusari, S. arXive: 1106.0437, 3 [cond-mat.mtrl-sci] (2012).

[17] Kopelevich, Y., Esquinazi, P. Adv. Mater., 19, 4559-4563 (2007).

[18] Barzola-Quiquia J., Ballestar A., Dusari S., Esquinazi P. Experimental Study of the Intrinsic and Extrinsic Transport Properties of Graphite and Multigraphene Samples; Graphene - Synthesis, Characterization, Properties and Applications,Gong J. (Ed.), pp. 115-140, 2011.

[19] Miyazaki H., Tsukagoshi K., Odaka S., Aoyagi Y., Sato T., Tanaka S., Goto H., Kanda A., Ootuka Y.Applied Physics Express 1034007 (2008).

[20] Ferrari A.C., Basko D.M. Nature Nanotechnology, 8, 235-246 (2013).

[21] Ferrari A.C., Meyer J.C., Scardaci C., Casiraghi C., Lazzeri, M., Mauri F., Piscanec S., Jiang D., Novoselov K.S., Rothe S., Geim A.K. Phys. Rev. Let., 97, 187401 (2006).

[22] Hao Y., Wang Y., Wang L., N,I. Zh., Wang Z., Wang R., Koo Ch.K., Shen Z., Thong J.T.L. Small, 6, 2, 195-200 (2010).

[23] ShmavonyanG.Sh., Giordani, S. Carbon Nanoscience and Nanotechnology Conference (NanoteC12), Brighton, UK (2012).

[24] Shin J.Y., Stromberg R., Nay R., Huang H., Wee A.T.S., Yang H., Bhatia Ch.S. Carbon 49, 4070-4073 (2011).

[25] Smolyanitsky A., Killgore J.P., Tewary V.K. Physical Review B 85, 035412 (2012).

[26] Bao Q.,Zhang H.,Wang B. Ni,Zh., Lim C.,Wang Yu, Tang D.Y.,Loh K.,P,Nature Photonics 5, 411-415 (2011).

[27] Thomas A.G., Fasolka M.J.Proc. SPIE 5188, 264-275 (2003). 